\title{
La révolution isocinétique
}

\section{Isocinetick revolution}

\author{
P. Middleton \\ C) Springer-Verlag France 2013
}

Conçus par des ingénieurs de la NASA, les dynamomètres isocinétiques ont été très rapidement adoptés, à la fin des années 1960, par nos confrères d'outre-Atlantique. Comme souvent, il a fallu un certain temps pour que cette technologie arrive sur notre continent et envahisse, très progressivement, notre pays.

L'isocinétisme présentait, en effet, un double intérêt. Tout d'abord de proposer un outil d'évaluation dynamique, mais aussi un outil de renforcement musculaire intelligent grâce à l'asservissement de la résistance aux capacités du patient.

Pour les anciens, dont je fais partie, il s'agissait d'une véritable révolution. Fini le travail statique intermittent. Fini les ordonnances de rééducation interdisant le travail dynamique.

Mais qui dit nouvelle technologie dit abus. Il y en a eu, certes, quand certains ont voulu réaliser des diagnostics étiologiques de certaines anomalies de courbe. Mais qui dit nouvelle technologie dit également engouement et amélioration de nos connaissances.

C'est la deuxième révolution isocinétique avec la réhabilitation du travail musculaire excentrique qui, jus- qu'alors, jouissait d'une réputation de travail musculaire iatrogène.

Aujourd'hui, les dynamomètres isocinétiques sont présents dans la plupart des centres de rééducation et, également, dans certains cabinets de kinésithérapie de ville. Cela prouve l'intérêt des thérapeutes pour l'isocinétisme dans sa capacité à améliorer la prise en charge des patients. La réalisation d'un travail isocinétique n'est plus l'apanage des sportifs. Les patients neurologiques, la réadaptation cardiaque, toute la pathologie mécanique de l'appareil locomoteur bénéficient des possibilités offertes par cette technologie.

Cela ne pouvait qu'émouvoir nos tutelles qui, en accordant un tarif de prise en charge pour l'évaluation isocinétique après ligamentoplastie du ligament croisé antérieur du genou, ont amorcé la troisième révolution : la tarification des actes isocinétiques.

Conflit d'intérêt : L'auteur a participé à la réalisation de formations sur l'utilisation des dynamomètres isocinétiques pour la société Prothia 